\title{
Training in robotic thoracic surgery
}

\author{
Paul L. Linsky, Benjamin Wei \\ Division of Cardiothoracic Surgery, University of Alabama-Birmingham Medical Center, Birmingham, AL 35294, USA \\ Contributions: (I) Conception and design: PL Linsky; (II) Administrative support: B Wei; (III) Provision of study materials or patients: None; (IV) \\ Collection and assembly of data: None; (V) Data analysis and interpretation: None; (VI) Manuscript writing: All authors; (VII) Final approval of \\ manuscript: All authors. \\ Correspondence to: Benjamin Wei, MD. Division of Cardiothoracic Surgery, University of Alabama at Birmingham, 703 19th St S, ZRB 739, \\ Birmingham, AL 35294, USA.Email : bwei@uab.edu.
}

\begin{abstract}
The best way to teach robotic thoracic surgery is still being decided. New trainees, experienced video-assisted thoracoscopic surgery (VATS) surgeons, and predominantly open surgeons each have different needs when it comes to learning robotic surgery. The data shows that the learning curve and ability to learn robotics initially appears to be shorter and easier than surgeons learning VATS. Though the absolute best method for teaching is still under investigation, multiple centers have started to create systematic methods of teaching robotic surgery that increases resident autonomy while still protecting the patient.
\end{abstract}

Keywords: Robotic surgery; thoracic surgery; education; resident training; training; robotic training; robot assisted

Received: 06 June 2017; Accepted: 05 December 2017; Published: 04 January 2018.

doi: 10.21037 /jovs.2017.12.12

View this article at: http://dx.doi.org/10.21037/jovs.2017.12.12

\section{Introduction}

The advent of robotic surgery and its utility in thoracic surgery is a great advancement in the field. As with any new technology, the question of how best to teach it has been raised. With this technology, many aspects must be considered. The current robotic platform has many features that make it an ideal instrument for teaching. In fact, the robotic platform may likely to be superior to other modes of thoracic surgery when it comes to training. This may be true for all experience levels learning the techniques. In considering the audience, there are really three different groups of surgeons that must be considered, new trainees, video-assisted thoracoscopic surgery (VATS) trained/ proficient surgeons, and open surgeons. Though some aspects of training will be the same no matter the trainee, they each bring different experiences to the robot. Enhanced technology, with better visualization and dual consoles make training in robotic surgery an excellent tool and facile.

\section{Role of simulator}

Simulation is a very important aspect of training in robotics for thoracic surgery. The surgeon should know how to maneuver and use the robot before operating on a live patient. The advantage of simulation is not a new concept. It has been demonstrated in multiple fields of medicine, including cardiac surgery $(1,2)$. The role of simulation in robotics has even been studied in medical students (3). This study took 24 medical students and tested their robotic skills by using two of the exercises in the Fundamentals of Laparoscopic Surgery, peg transfer and intracorporeal knot tying. Initially, they were exposed to the tasks without any simulator training. After three attempts at each skill, they were scored. They all underwent robotic simulation training and were retested. All of the students reached proficiency in an average of 71 attempts per skill, which was only 164 minutes of simulator time.

At our institution, every resident must score $70 \%$ on 14 of the 30 exercises prior to being given permission to perform any operation. One weak aspect of the current simulator may be knot-tying. That skill can be practiced either with the current simulation software or with physical models. The models need set up with the help of staff from Intuitive. The models do require a complete $\mathrm{Da}$ Vinci system rather than the single 
console with a simulation package.

\section{Advantages of the system for teaching}

The Da Vinci systems have several clear advantages when it comes to training. The first is in the dual consoles. While training a novice, the experienced teacher not only has the same view as the trainee, but essentially has the same hands. If the trainee is having an issue with performing a task, especially from a conceptual point, the instructor can assume control of the robotic arms and show or perform the task with just a pressing of a few buttons on the console. This does not involve switching positions, which lengthens the operation, or performing the maneuver from the opposite side. The trainee can see directly, instantaneously, and in the same orientation how that step should be performed. The instructor can even use arrows to point to exactly the place he or she is trying the train to place an instrument or where an anatomical structure is located by using the controls of their consoles. There is even a drawing function on the main screen of the robot hub that can further enhance instruction. Not only does this make the robot a great platform for teaching, it ultimately provides an extra level of safety to the patient while the trainee is learning.

Secondly, the robotic platform brings with it the ease of recording operations. Every operation on the system can be recorded and reviewed at a later date. Though this is not impossible with open or VATS, it greatly improves the learning of the trainee by reviewing the recording, especially with the instructor. This is much like a player and a coach reviewing a game film. Hu, et al. from Brigham and Woman's performed a study where four different operations, complex in nature, were performed by surgeons of varying experience. The levels of experience were chief residents assisted by the operating senior attending surgeon, surgeons with under 10 years in practice, surgeons with between 20 and 30 years' experience, and, finally, a surgeon with more than 30 years. They were all video recorded (4). The videos were then reviewed by a peer selected expert and then a 1-hour video review session was held. Obviously, especially for younger surgeons, most of the discussion revolved around operative strategy and techniques. Interestingly, with increasing seniority, the session was more directed to the teaching styles of the surgeon, with the goal being the improvement of the resident's technical skills. They concluded that video-based coaching improves intraoperative judgment, technique, and teaching. All the surgeons, despite their level of experience, found the experience very rewarding. Ultimately, they felt that video-based coaching may provide a easily reproducible means and needed technique for continuing professional education and skill development. The robotic platform is ideal for this strategy.

\section{Learning robotic thoracic surgery}

When beginning in robotic thoracic surgery, we do not advocate attempting to complete the entire procedure robotically, especially complex surgeries such as lobectomy. Each operation has a sequence of steps of increasing difficulty and the surgery can easily be divided into these steps. The surgeon should focus on completing the procedure in a step wise fashion, by performing and completing the more basic parts of the procedure before advancing onto more difficult parts. Each thoracic procedure can be given a level, as shown in Table 1 . Surgeons should start with Level I operations before advancing to higher level operations.

Using a lobectomy as an example, Cerfolio et al., lays a sequence for surgeons to learn how to perform a robotic lobectomy. On the first lobectomy, the surgeon may only place trocars, dock the robot, and perform a mediastinal lymphadenectomy. At this point, the surgeon electively converts to VATS or open techniques for the pulmonary resection. With each operation, the surgeon builds on his or her previous experience by not only completing the same steps robotically, but doing more steps with the robot.

In their experience, the steps that will be done robotically should be determined prior to the operation. In doing so, the operating room (OR) team understands what is going to be done, so that they can be prepared and so they do not lose confidence in the transition to robotics. The importance of the team being a part of the surgery is crucial to good outcomes, shorter operative times, and the success of the robotics program.

\section{Surgeons teaching robotics}

When teaching robotic thoracic surgery, a step-wise progression can be used no matter what the level of experience of the trainee. The key difference is that trained surgeon can finish the operation robotically without going to VATS or open. This allows the trainee to see what needs to be done. With more training and attempts, the trainee can attempt to perform more of the operation.

As with any operation, the "set-up" of an operation is crucial to completing the operation. That specifically 
Table 1 Classification of Level I, II and III operations for thoracic surgeons

\begin{tabular}{|c|c|c|c|c|c|}
\hline \multicolumn{2}{|c|}{ Level I operations } & \multicolumn{2}{|c|}{ Level II operations } & \multicolumn{2}{|c|}{ Level III operations } \\
\hline$*$ & $\begin{array}{l}\text { Resection of esophageal or pulmonary } \\
\text { mediastinal cyst }\end{array}$ & $*$ & $\begin{array}{l}\text { Resection of inferior (lower than 9th rib) } \\
\text { posterior mediastinal tumors }\end{array}$ & $*$ & Lobectomy \\
\hline$*$ & $\begin{array}{l}\text { Wedge resection of lung, not directed for a } \\
\text { specific nodule* }\end{array}$ & $*$ & Leiomyoma of mid-esophagus & $*$ & $\begin{array}{l}\text { Myotomy of esophagus } \\
\text { for achalasia with Dor } \\
\text { fundoplication }\end{array}$ \\
\hline$*$ & $\begin{array}{l}\text { Wedge resection of lung, directed for a specific } \\
\text { nodule* }^{*}\end{array}$ & $*$ & Leiomyoma of mid-esophagus & $*$ & $\begin{array}{l}\text { Robotic Ivor Lewis } \\
\text { esophagectomy }\end{array}$ \\
\hline$*$ & Pleural biopsy* & & & & \\
\hline
\end{tabular}

*, Vats may be preferred technique but reasonable to use robot for training to increase robotic experience.

includes port positioning and retraction of structures. Emphasizing "set-up" will allow for the trainee to more able to be successful sooner in his or her experience. Additionally, using the dual consoles lets the teacher to see exactly how the trainee uses the console which is critical to being able to reduce the trainee's operative times. The training surgeon can really expedite the progress of the trainee by doing this.

\section{Established surgeons learning robotics}

As with any new technology, the question is raised about how many procedures are needed to be performed to be proficient when switching technology. Different studies report different results. Across the literature, the number seems to be around 20 cases (5-9). Lee et al. reported that in their initial robotic lobectomy experience that they found no difference in operative times when performing lower lobectomies when compared to mature VATS surgeons, but that there was a difference in the operative times for upper lobectomy (10). They felt that the learning curve was 17 cases, which is consistent with most of the other studies in the literature.

Jang et al. cite the lowest learning curve at 6 cases (11). In their study, they compared their initial robotic lobectomies to their initial VATS and current VATS outcomes. They found their robotic experience superior to both the initial and current VATS experience. They reported no conversions to open thoracotomy, but conversion rates were $8 \%(3)$ and $5 \%$ (2) in their early and late VATS patients, respectively. The operative time for the robotic group was slightly shorter than their initial VATS lobectomy groups

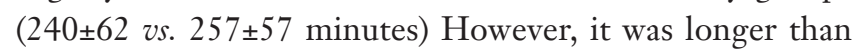

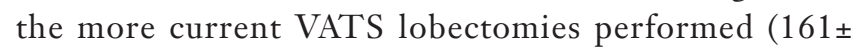
39 minutes). Of note, the postoperative complications rate for their robotic experience was lower than that from their early VATS experience and equivalent to their most recent VATS group $(n=4,10 \% ; n=13,32.5 \% ; n=7,17.5 \%$, respectively). In regards to bleeding, the robotic group was more than $155 \mathrm{~mL}$ less than the initial VATS group (219 vs. $374 \mathrm{~mL}, \mathrm{P}=0.017)$. In their study, postoperative stay was reduced in the robotic group compared to their VATS group (6 vs. 9 days, $\mathrm{P}<0.001$ ).

Most of the literature focuses on converting a practice from VATS to robotics. However, there are some studies to suggest that robotics may be an easier transition from open surgery than to convert to VATS first. One study reported the learning curve for VATS was 30 cases for safety and 90 for efficiency (12). One paper even expanded that number to 100-200 for safety and efficiency and more for excellence (13). Overall, most agree the number is 30 or more, or 6 to 12 months of experience (14-16). 
A unique study was performed comparing the skills of 8 attending surgeons on intracorporeal knot tying on the robot compared to laparoscopically (17). A scoring system was used to evaluate the exercise and the participants were tested over a 3 week period. The surgeons were initially scored and they then had 3 weeks of training on a surgical robot. Their knots made robotically were compared to those done laparoscopically. The knots were analyzed to determine skill improvement. The laparoscopic knots were completed in $140 \mathrm{sec}$ with a range of $47-432 \mathrm{secs}$, with a mean composite score of 77.100 was the highest possible score. The robotic knots took $390 \mathrm{sec}$ to complete, with a mean composite score of 40 . After the 3 weeks of robotic training, the times decreased $65 \%$ to 139 secs and scores went up to 71 . As one would expect, with further training, times to complete and scores improved and less errors occurred. The authors concluded surgeons can become more proficient with robotic skills, making better knots and doing them faster if they receive the appropriate training. Also, they stated that even experienced laparoscopists may struggle with a new technology, and mastery of robotic surgery requires dedicated training.

\section{Resident training for robotics}

In a recent report from the Society of Thoracic Surgeons Workforce on Thoracic Surgery Resident Issues Transition to Practice Task Force, recent graduates reported in a survey that the three areas of most needed improvement by exposure in training programs was practice management, congenital cardiac surgery and robotic procedures (18). Of particular note, the responders felt that they lacked confidence in robotic pulmonary operations (29/52, 55.8\%) and robotic esophageal operations (32/52, 61.5\%). Along with congenital cardiac and robotic adult cardiac cases, these were the highest responses of a clinical nature. This shows that new graduates feel that they need more instruction in robotics during their training. This is not unique to thoracic surgery. In a survey sent to general surgery residents, most responded that they had assisted in 10 or fewer robotic cases with the most frequent activities being assisting with robotic trocar placement and docking and undocking the robot (19). Less than $20 \%$ reported operating from the robotic console. Resident involvement increased with seniority. Of those in the study, $64 \%$ felt robotic training was important in residency training.

As with any training program, the analysis of patient outcomes has been examined. Of course, the safety and well- being of the patient take precedent over education. Several studies have been performed assessing risk of training residents on the robotic platform. White et al., from the University of Michigan reported their experience during their first 100 thoracic robot cases (20). Of those cases, just under $40 \%$ were lung resections, one quarter were esophageal operations, and one fifth were sympathectomies. Additionally, during these first 100 cases, the percentage of residents acting as the primary surgeon doubled from 33\% to $59 \%(\mathrm{P}<0.05)$. When analysis of the lobectomies was performed, with 7 being performed by attendings and 13 by residents, outcomes were similar for intraoperative and postoperative parameters such as operative time (260 vs. $249 \mathrm{~min}$ ), estimated blood loss (187 vs. $203 \mathrm{~mL}$ ), and length of stay ( $4.8 v s .4 .7 \mathrm{~d})$.

The Mayo Clinic reported their experience in robotic training over a 4-year period. During that time, 79 procedures were robot-assisted (21). The study included one attending and three residents. They participated in a triphasic, competency-based pathway for learning skills robotically. The pathway consisted of with participants performing individual learning prior to any clinical activity. That was the followed by mentored preclinical exercises and finally with progressive clinical responsibility. During this period, there was a $3 \%$ readmission rate and no perioperative mortalities. However, there was a $20 \%$ complication rate. Only eight procedures were converted to VATS or open approach, but only two were to control hemorrhage. Major perioperative complications decreased in the latter two years. The most significant finding about the study is that all residents who participated went on to perform robotic procedures in their clinical practice.

More recently, Cerfolio et al., reported their experience on resident training at the University of Alabama (22). Over a 5 -year period, 520 consecutive robotic lobectomies with various parts of the operation being performed by general surgical residents $(\mathrm{N}=35)$ and cardiothoracic residents $(\mathrm{N}=7)$. During that time period each of the steps were performed by the trainees with no change in operative outcomes, including intraoperative blood loss, median number of lymph nodes, median length of stay, major morbidity, and 30-day and 90-day mortality. Operative time initially increased and then decreased over time. Additionally in that paper, he lays out a step wise progression of how to teach a thoracic resident. Table 2 comes from that work and shows the steps and time expectations on those performing the operation. 
Table 2 The recorded sequential steps of each lobectomy (in order of conduct) and allotted time to be completed

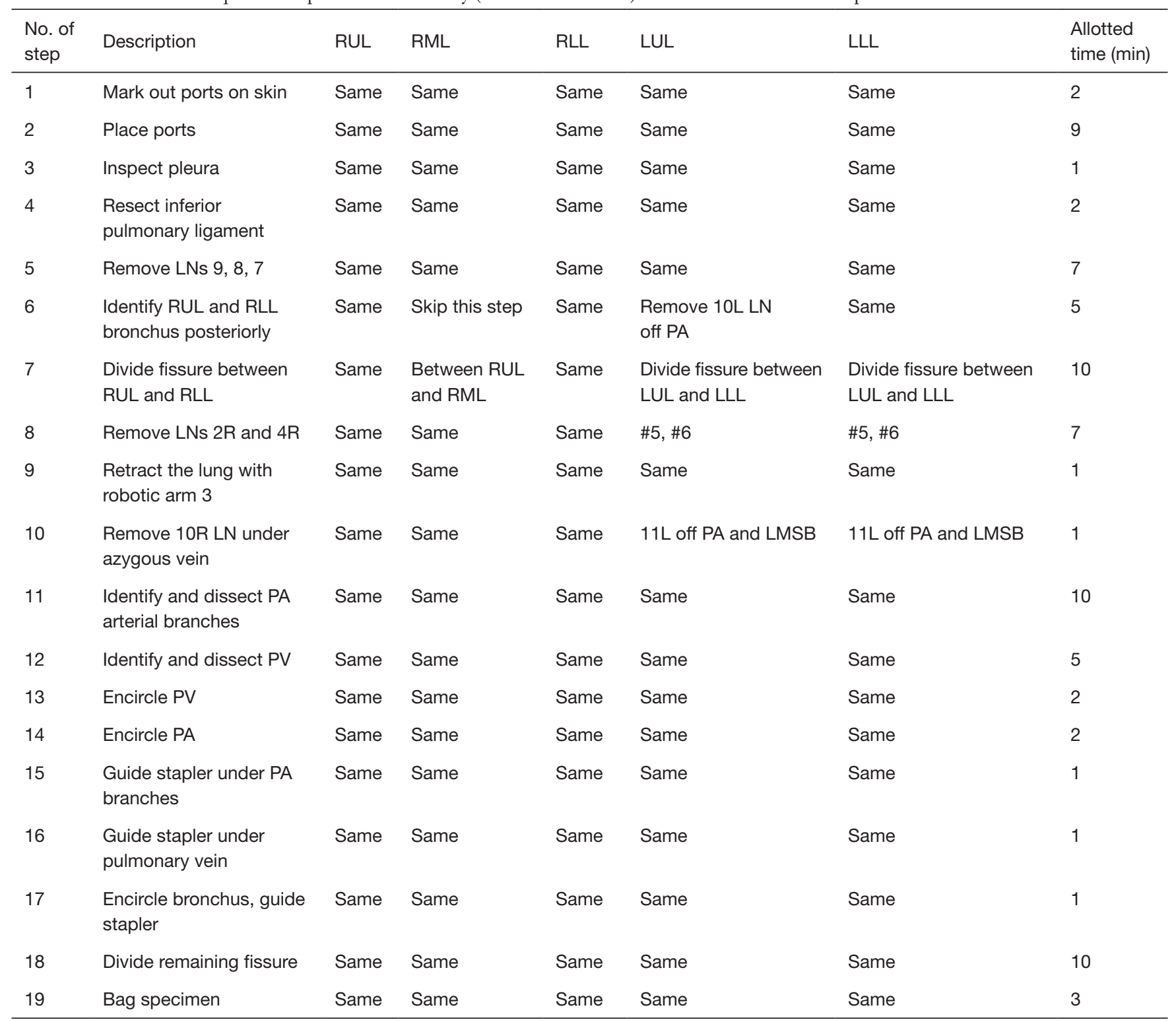

RUL, right upper lobe; RML, right middle lobe; RLL, right lower lobe; LUL, left upper lobe; LLL, left lower lobe; LN, lymph node; PA, pulmonary artery; LMSB, left main stem bronchus; PV, pulmonary vein.

\section{Conclusions}

As with any new technology, robotic surgery must be taught to new users. Different techniques and systems need to be developed and used to train surgeons of different skill levels. However, the design of the current systems gives teaching robotic thoracic surgery several key advantages that likely give it an advantage over open or VATS. This advantage will benefit the instructor, the trainee, and, most importantly, the patient. There is some evidence that learning robotics may be easier than learning VATS. Ultimately, a curriculum will be needed to train and credential surgeons in robotics. As robotic thoracic surgery evolves, so will its teaching.

\section{Acknowledgements}

None. 


\section{Footnote}

Conflicts of Interest: The authors have no conflicts of interest to declare.

\section{References}

1. Valdis M, Chu MW, Schlachta CM, et al. Validation of a novel virtual reality training curriculum for robotic cardiac surgery: a randomized trial. Innovations (Phila) 2015;10:383-8.

2. Valdis M, Chu MW, Schlachta C, et al. Evaluation of robotic cardiac surgery simulation training: A randomized controlled trial. J Thorac Cardiovasc Surg 2016;151:1498-505.e2.

3. Bric J, Connolly M, Kastenmeier A, et al. Proficiency training on a virtual reality robotic surgical skills curriculum. Surg Endosc 2014;28:3343-8.

4. Hu YY, Peyre SE, Arriaga AF, et al. Postgame analysis: using video-based coaching for continuous professional development. J Am Coll Surg 2012;214:115-24.

5. Yamashita S, Yoshida Y, Iwasaki A. Robotic surgery for thoracic disease. Ann Thorac Cardiovasc Surg 2016;22:1-5.

6. Gharagozloo F, Margolis M, Tempesta B, et al. Robotassisted lobectomy for early-stage lung cancer: report of 100 consecutive cases. Ann Thorac Surg 2009;88:380-4.

7. Melfi FM, Mussi A. Robotically assisted lobectomy: learning curve and complications. Thorac Surg Clin 2008;18:289-95, vi-vii.

8. Veronesi G, Agoglia BG, Melfi F, et al. Experience with robotic lobectomy for lung cancer. Innovations (Phila) 2011;6:355-60.

9. Meyer M, Gharagozloo F, Tempesta B, et al. The learning curve of robotic lobectomy. Int J Med Robot 2012;8:448-52.

10. Lee BE, Korst RJ, Kletsman E, et al. Transitioning from video-assisted thoracic surgical lobectomy to robotics for lung cancer: are there outcomes advantages? J Thorac Cardiovasc Surg 2014;147:724-9.

11. Jang HJ, Lee HS, Park SY, et al. Comparison of the early

doi: 10.21037/jovs.2017.12.12

Cite this article as: Linsky PL, Wei B. Training in robotic thoracic surgery. J Vis Surg 2017;4:1. robot-assisted lobectomy experience to video-assisted thoracic surgery lobectomy for lung cancer: a singleinstitution case series matching study. Innovations (Phila) 2011;6:305-10.

12. Mazzella A, Olland A, Falcoz PE, et al. Video-assisted thoracoscopic lobectomy: which is the learning curve of an experienced consultant? J Thorac Dis 2016;8:2444-53.

13. Li X, Wang J, Ferguson MK. Competence versus mastery: the time course for developing proficiency in videoassisted thoracoscopic lobectomy. J Thorac Cardiovasc Surg 2014;147:1150-4.

14. Arad T, Levi-Faber D, Nir RR, et al. The learning curve of video-assisted thoracoscopic surgery (VATS) for lung lobectomy--a single Israeli center experience. Harefuah 2012;151:261-5, 320.

15. Ra YJ, Ahn HY, Kim MS. Learning curve of a young surgeon's video-assisted thoracic surgery lobectomy during his first year experience in newly established institution. Korean J Thorac Cardiovasc Surg 2012;45:166-70.

16. Zhao H, Bu L, Yang F, et al. Video-assisted thoracoscopic surgery lobectomy for lung cancer: the learning curve. World J Surg 2010;34:2368-72.

17. Chang L, Satava RM, Pellegrini CA, et al. Robotic surgery: identifying the learning curve through objective measurement of skill. Surg Endosc 2003;17:1744-8.

18. Chu D, Vaporciyan AA, Iannettoni MD, et al. Are there gaps in current thoracic surgery residency training programs? Ann Thorac Surg 2016;101:2350-5.

19. Farivar BS, Flannagan M, Leitman IM. General surgery residents' perception of robot-assisted procedures during surgical training. J Surg Educ 2015;72:235-42.

20. White YN, Dedhia P, Bergeron EJ, et al. Resident training in a new robotic thoracic surgery program. J Surg Res 2016;201:219-25.

21. Seder CW, Cassivi SD, Wigle DA. Navigating the pathway to robotic competency in general thoracic surgery. Innovations (Phila) 2013;8:184-9.

22. Cerfolio RJ, Cichos KH, Wei B, et al. Robotic lobectomy can be taught while maintaining quality patient outcomes. J Thorac Cardiovasc Surg 2016;152:991-7. 